\title{
Effect of Disorder on the Quantum Coherence in Mesoscopic Wires
}

\author{
Y. Niimi ${ }^{1, \dagger}$, Y. Baines ${ }^{1}$, T. Capron ${ }^{1}$, D. Mailly ${ }^{2}$, F.-Y. Lo ${ }^{3, \ddagger}$, \\ A. D. Wieck ${ }^{3}$, T. Meunier ${ }^{1, *}$, L. Saminadayar ${ }^{1,4,5, *}$ and C. Bäuerle ${ }^{1, *}$ \\ ${ }^{1}$ Institut Néel, CNRS, B.P. 166, 38042 Grenoble Cedex 09, France \\ ${ }^{2}$ Laboratoire de Photonique et Nanostructures, route de Nozay, 91460 Marcoussis, France \\ ${ }^{3}$ Lehrstuhl für Angewandte Festkörperphysik, Ruhr-Universität, \\ Universitätsstraße 150, 44780 Bochum, Germany \\ ${ }^{4}$ Université Joseph Fourier, B.P. 53, 38041 Grenoble Cedex 09, France and \\ ${ }^{5}$ Institut Universitaire de France, 103 boulevard Saint-Michel, 75005 Paris, France
}

(Dated: 1 June 2009)

\begin{abstract}
We present phase coherence time measurements in quasi-one-dimensional mesoscopic wires made from high mobility two-dimensional electron gas. By implanting gallium ions into a GaAs/AlGaAs heterojunction we are able to vary the diffusion coefficient over 2 orders of magnitude. We show that in the diffusive limit, the decoherence time follows a power law as a function of diffusion coefficient as expected by theory. When the disorder is low enough so that the samples are semi-ballistic, we observe a new and unexpected regime in which the phase coherence time is independent of disorder. In addition, for all samples the temperature dependence of the phase coherence time follows a power law down to the lowest temperatures without any sign of saturation and this strongly suggests that the frequently observed low temperature saturation is not intrinsic.
\end{abstract}

PACS numbers: 73.23.-b, 73.63.Nm, 03.65.Yz

Keywords:

Decoherence is the process by which a quantum system looses phase coherence by getting entangled with its environment. For a metallic system, the dominant mechanisms for decoherence of electrons are inelastic scattering events due to collisions with other electrons, phonons, and also extrinsic sources such as magnetic impurities or two level systems. Whereas at temperatures above a few Kelvin the electron-phonon interactions are dominant, at low temperatures and without extrinsic sources of decoherence, the leading decoherence process is due to electron-electron interactions and has been at the center of a controversy over the last decade: the "standard" theory of electron coherence in metals by Altshuler, Aronov and Khmelnitski (AAK) [1] predicts a diverging dephasing time with vanishing temperature, while an alternative theory proposed by Golubev and Zaikin (GZ) predicts a saturation of the dephasing time at low temperatures [2]. Saturation of the dephasing time down to absolute zero raises the question of the breakdown of the Fermi liquid theory and, as such, is of basic importance for our understanding of metallic conductors.

The experiments by Mohanty et al. 3], showing a systematic saturation of the phase coherence time $\tau_{\phi}$ at low temperature have triggered a large amount of studies, both from experimental and theoretical points of view. These studies aimed to show whether the saturation of $\tau_{\phi}$ at low temperature is either intrinsic [2, 3], or due to any other extrinsic mechanisms [4, 5, 6, 7, 8, 9, 10]. It should be stressed, however, that for all these experiments only the temperature dependence of $\tau_{\phi}$ has been investigated. On the other hand, it has been shown that this approach is by far insufficient, as the presence of a tiny amount of magnetic impurities with given Kondo temperature can always account for the experimentally observed saturation of $\tau_{\phi} 11,12,13$. It is thus utterly important to find another parameter to be able to discriminate between the different scenarios.

It is our purpose to break this impasse by taking a new approach. Instead of studying the temperature dependence of $\tau_{\phi}$ we exploit another property which should shine new light on this problem and which has been almost unexplored so far: the dependence of the phase coherence time on the disorder, or, in other words, on the diffusion coefficient $D$. Some attempts to measure this dependence have been realized in metallic systems [14, 15], but in this case it is very difficult to vary $D$ in a controlled way over a wide range. Another study has been performed on two-dimensional electron systems [16], but in that work the phase coherence time was already saturating at $2 \mathrm{~K}$ in their cleanest samples. Those works thus do not allow to draw any clear conclusions about the dependence of the phase coherence on disorder.

In this Letter, we report on measurements of the electronic phase coherence time $\tau_{\phi}$ in wires made from a twodimensional electron gas (2DEG) where the diffusion coefficient $D$ is varied in an extremely controlled way over 2 orders of magnitude by using an original ion implantation technique. In the diffusive regime, the $D$ dependence of the phase coherence time $\tau_{\phi}$ follows a power law $D^{\alpha}$ with $\alpha$ close to $1 / 3$. More surprisingly, when entering the semi-ballistic regime, the phase coherence time becomes independent of disorder. Moreover, for all samples the temperature dependence of $\tau_{\phi}$ follows a power law down to the lowest temperature without any sign of saturation; this casts serious doubt that the frequently observed low temperature saturation could be intrinsic. 
The difficulty with measurements of the decoherence in any metal is the purity of the metal source. A careful choice of the starting material is of utmost importance as any tiny inclusion of impurities could lead to an apparent saturation at low temperatures [13]. For this reason we have used a high mobility GaAs/AlGaAs heterostructure as starting material. These systems are intrinsically clean and grown in ultrahigh vacuum by molecular beam epitaxy. Before processing, the 2DEG has an electron density of $n_{e}=1.76 \times 10^{11} \mathrm{~cm}^{-2}$ and mobility $\mu=1.26 \times 10^{6} \mathrm{~cm}^{2} / \mathrm{V} \cdot \mathrm{s}$ at $4 \mathrm{~K}$ in the dark. All lithographic steps are performed using electron beam lithography on polymethyl-methacrylate resist. Each sample (see inset of Fig. 2) consists of 4 sets of 20 wires of length $L=150 \mu \mathrm{m}$ in parallel, and of lithographic width $W=600,800,1000$ and $1500 \mathrm{~nm}$. In addition, a Hall bar allows us to measure the electronic parameters of the 2DEG (i.e. $n_{e}, \mu$, the elastic mean free path $l_{e}$, etc.). The diffusion coefficient is obtained via the relation $D=v_{F} l_{e} / 2$ with $v_{F}$ the Fermi velocity. In order to change the diffusion coefficient of our samples we use a very original technique: first we write the pattern of each sample on the same wafer. We then place a Focused Ion Beam (FIB) microscope on one sample and implant locally $\mathrm{Ga}^{+}$ions with an energy of $100 \mathrm{keV}$. For this energy, the $\mathrm{Ga}^{+}$ions penetrate only about $50 \mathrm{~nm}$ into the GaAs heterostructure, whereas the 2DEG lies $110 \mathrm{~nm}$ below the surface. The implanted ions create crystal defects in the AlGaAs layer and modify the intrinsic disorder potential felt by the electrons; note, however, that in the case of low dopings like here, the band structure is not changed [17]. This affects only the momentum scattering time and thus the mobility of the itinerant electrons in the 2DEG [18]. By varying the implantation dose for different samples $\left(10^{8} \mathrm{~cm}^{-2}\right.$ to $\left.2.5 \times 10^{9} \mathrm{~cm}^{-2}\right)$ we are able to vary the diffusion coefficient from $3500 \mathrm{~cm}^{2} / \mathrm{s}$ (unimplanted sample) to $130 \mathrm{~cm}^{2} / \mathrm{s}$. Magnetoconductance measurements are performed using a standard ac lock-in technique and a home-made very low noise preamplifier $(0.5 \mathrm{nV} / \sqrt{\mathrm{Hz}})$ at room temperature. The temperature dependence of the resistivity is measured on the Hall bar and used to check the actual temperature of the electrons of the sample: we find that it follows nicely a $\ln (T)$ law down to a temperature of $40 \mathrm{mK}$ as expected [1]. Below this temperature the electrons decouple slightly from the base temperature of our dilution refrigerator; we then use the resistivity of the Hall bar as the thermometer, assuming that the $\ln (T)$ law holds down to the lowest temperature.

Depending on the number of impurities different scattering regimes are accessible: the ballistic regime $\left(l_{e}>\right.$ $W, L)$, the semi-ballistic regime $\left(W<l_{e}<L\right)$ and the diffusive regime $\left(l_{e}<W, L\right)$. The latter two regimes have been explored in this work.

Diffusive regime: When $l_{e}<W$, the scattering of the electrons is diffusive. In this regime, the weak localization

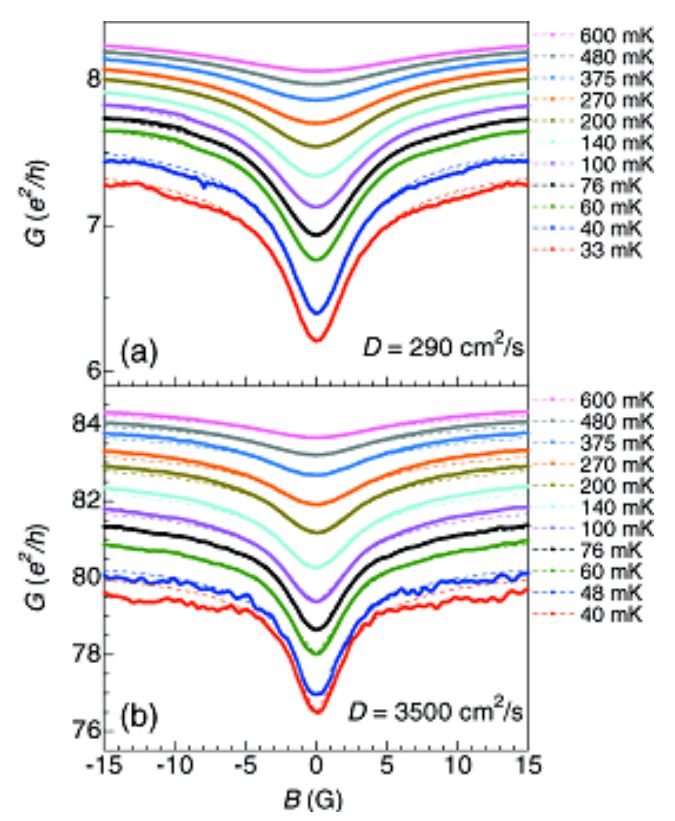

FIG. 1: (Color online) Magnetoconductance curves of 2DEG wires with $w=1130 \mathrm{~nm}$ for (a) $D=290 \mathrm{~cm}^{2} / \mathrm{s}\left(l_{e}=0.34 \mu \mathrm{m}\right)$ and (b) $3500 \mathrm{~cm}^{2} / \mathrm{s}(4.0 \mu \mathrm{m})$ at different temperatures. The broken lines show theoretical fits to weak localization theory for (a) the diffusive and (b) the semi-ballistic regimes.

quantum correction to the conductance is given by [1, 19]

$$
\Delta G(B)=-2 \frac{e^{2}}{h} \frac{N}{L}\left\{\frac{1}{\sqrt{\frac{1}{L_{\phi}^{2}}+\frac{w^{2}}{3 l_{B}^{4}}}}\right\},
$$

where $e^{2} / h$ is the quantum of conductance, $(e$ is the charge of the electron, $h$ the Planck's constant), $L_{\phi}$ the phase coherence length, $N$ the number of wires in parallel $(N=20$ in the present work) and $w$ is the effective width of the wires, different from the lithographic width $W$ due to lateral depletion effects inherent to the etching process. We therefore determine $w$ by fitting the magnetoconductance at a given temperature and diffusion coefficient: for a lithographic width $W=1500 \mathrm{~nm}$ we obtain $w=1130 \mathrm{~nm}$. This value is then kept fixed for the entire fitting procedure.

Semi-ballistic regime $\left(W<l_{e}\right)$ : In this regime it is necessary to take into account specular reflections on the boundary of the wires and flux cancelation effects. The weak localization correction has been calculated by Beenakker and van Houten (BvH) [20] and are given below:

$\Delta G(B)=-2 \frac{e^{2}}{h} \frac{N}{L}\left\{\frac{1}{\sqrt{\frac{1}{L_{\phi}^{2}}+\frac{1}{D \tau_{B}}}}-\frac{1}{\sqrt{\frac{1}{L_{\phi}^{2}}+\frac{1}{D \tau_{B}}+\frac{2}{l_{e}^{2}}}}\right\}$

In the magnetic scattering time $\tau_{B}$, both the "low" field and "high" field regimes are taken into account via the 


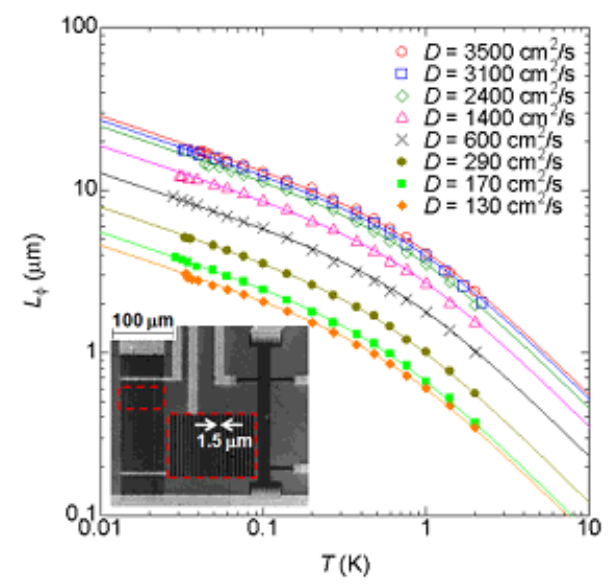

FIG. 2: (Color online) Phase coherence length $L_{\phi}$ for different $D$ as a function of $T(w=1130 \mathrm{~nm})$. The solid lines are the best fits with Eq. (3) adding an extra term $\propto T^{-1}$ for high energy processes [16]. The open and closed symbols are used for the semi-ballistic and diffusive regimes, respectively. The inset shows 2DEG wires with $W=1500 \mathrm{~nm}$ and a Hall bar.

expression

$$
D \tau_{B}=D \tau_{B}^{\text {low }}+D \tau_{B}^{\text {high }}=\frac{9.5}{2} \frac{l_{B}^{4} l_{e}}{w^{3}}+\frac{4.8}{2} \frac{l_{B}^{2} l_{e}^{2}}{w^{2}} .
$$

As $l_{e}$ is known from independent measurement on the Hall bar sample, $L_{\phi}$ remains the only fitting parameter for both regimes.

Typical magnetoconductance curves for the diffusive and semi-ballistic samples with diffusion coefficients $D=$ 290 and $3500 \mathrm{~cm}^{2} / \mathrm{s}$ are displayed in Figs. 1(a) and 1(b), respectively. Fitting the experimental data to Eqs. (1D) and (2), dashed lines in Fig. 1, we can extract the phase coherence length $L_{\phi}$ for each temperature. Note, however, that these expressions are valid only in a regime where the magnetic length $l_{B}=\sqrt{\hbar / e|B|}$ is larger than the effective width of the wire $w$; we thus restrict our magnetic field range for fitting procedure to a field range of $\pm 5 \mathrm{G}$. The observed deviations above $5 \mathrm{G}$ in Fig. 1 (b) are consistent with the condition of validity of the $\mathrm{BvH}$ expression [21].

The temperature dependence of the phase coherence length $L_{\phi}$ is displayed in Fig. 2 for samples with several different diffusion coefficients. At low temperatures the dominant decoherence mechanism is due to electronelectron interactions and the phase coherence time for quasi-1D diffusive wires is given by [1, 19]

$$
\begin{aligned}
\frac{1}{\tau_{\phi}}=\frac{D}{L_{\phi}^{2}} & =a T^{2 / 3} \\
& \equiv \alpha_{\mathrm{AAK}} D^{-1 / 3} T^{2 / 3}
\end{aligned}
$$

where $\alpha_{\mathrm{AAK}}=1 / 2\left(k_{B} \pi / w m^{*}\right)^{2 / 3}$ with $k_{B}$ the Boltzmann constant and $m^{*}$ the effective mass of the elec-

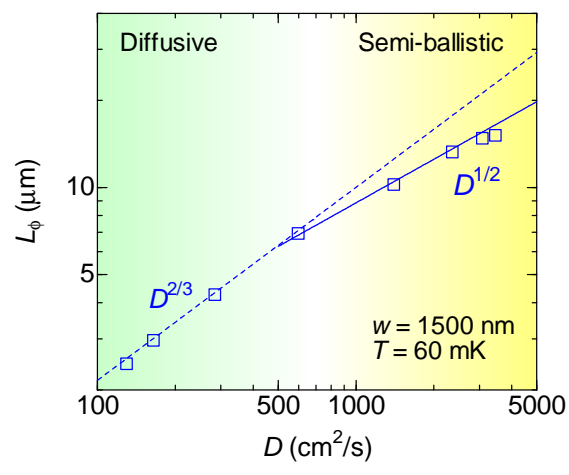

FIG. 3: (Color online) Phase coherence length $L_{\phi}$ as a function of $D(w=1130 \mathrm{~nm})$ at a temperature of $T=60 \mathrm{mK}$. The solid and broken lines represent $D^{1 / 2}$ and $D^{2 / 3}$ laws, respectively.

tron. As shown in Fig. 2, for all the investigated samples, $L_{\phi}(T)$ is described very well by Eq. (3), where a is treated as a fitting parameter $\left(a_{\exp }\right)$, and adding an extra term $\propto T^{-1}$ for "high" energy processes 16].

We can go further in the analysis by looking at the $L_{\phi}$ dependence as a function of $D$. Let us remind that the diffusion coefficient reflects the strength of the electronelectron interaction [1, 19] and thus controls the low temperature behavior of the dephasing rate. In Fig. 3 we plot $L_{\phi}$ as a function of $D$ at a temperature of $60 \mathrm{mK}$. One clearly observes two different regimes: For small $D$, $L_{\phi}$ varies as a power law $D^{\gamma}$ with $\gamma \approx 2 / 3$, whereas for large $D, \gamma \approx 1 / 2$. In order to compare more precisely the $D$ dependence of the prefactor $a$ in Eq. (3) with theoretical predictions, we have plotted in Fig. 4 the experimental parameter $a_{\exp }$ extracted from the fits depicted on Fig. 2. normalized by the theoretical prefactor $\alpha_{\mathrm{AAK}}$ and as a function of $D$. To compute $a_{\exp }$ we use the relation $L_{\phi}=\sqrt{D \tau_{\phi}}$, which is valid in the diffusive limit as well as in the semi-ballistic one when reflections on the boundaries are specular (as is the case for our samples) [20, 22]. Furthermore, we have checked that the resistivity for all our samples is linear with $D$ : this fact also justifies the above assumption. In the diffusive regime (small $D$ ), we obtain that the parameter $a_{\exp } / \alpha_{\mathrm{AAK}}$ follows a power law as a function of $D$, with $a_{\exp } / \alpha_{\mathrm{AAK}} \propto D^{-1 / 3}$, consistent with Eq. (4). Moreover, the prefactor $a_{\exp }$ obtained in this work agrees with Eq. (4) in absolute value within $15 \%$.

Surprisingly, a new regime appears when we go further in the "metallic" regime: when $k_{F} l_{e}$ increases [see lower part of Fig. 4, the samples become semi-ballistic. In this regime, the parameter $a_{\exp } / \alpha_{\mathrm{AAK}}$ becomes independent of the diffusion coefficient. What could be the origin of this disorder independent decoherence? One could argue that for such high mobility samples the scattering on the boundaries becomes dominant: if the boundary scattering of the electrons is diffusive, the diffusion coefficient 


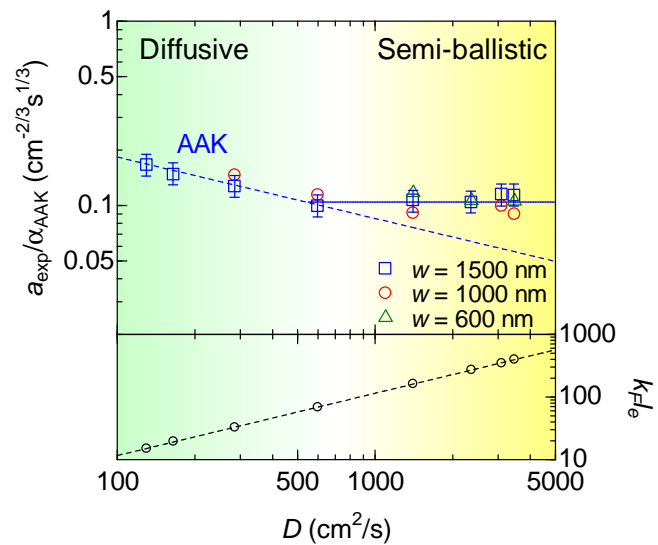

FIG. 4: (Color online) Top figure shows the experimental coefficients $a_{\exp }$ of Eq. (3) scaled by $\alpha_{\mathrm{AAK}}$ as a function of $D$. The dashed line represents $D^{-1 / 3}$. The experimental values of $k_{F} l_{e}$ are shown in the bottom part of the figure.

should eventually saturate at $D=1 / 2 v_{F} w[20]$ and could lead to disorder-independent decoherence. This possibility, however, is easily ruled out. Our samples have been made by shallow etching and the reflections on the boundaries are mainly specular 22]. In addition, the resistance of all our samples varies linearly with $D$. On the other hand, it is remarkable that the temperature dependence of $L_{\phi}$ follows extremely well the power law predicted in 1] even in this semi-ballistic regime 23]. This shows that the role of the temperature in the decoherence process is well captured in the theory; the role of the disorder, on the other hand, has to be reconsidered to describe properly our experimental observations. To our knowledge, there is only one theoretical attempt to consider this regime where the amplitude of the weak localization is reduced with increasing $l_{e}$ [24], but this calculation has been performed (numerically) for a twodimensional system and only at two typical values of $l_{e}$ and hence does not allow for a quantitative analysis of our experimental data.

Finally, let us mention that in the GZ theory, the low temperature behavior of the decoherence time $\tau_{\phi}$ should strongly depend on $D$ [2]. In particular $\tau_{\phi}(T)$ is expected to saturate at zero temperature at a finite value $\tau_{\phi}^{0}$ which itself should depend very strongly on $D$. Such a saturation is not observed for any of our samples for all diffusion coefficients investigated. This is corroborated by the fact that for decoherence measurements in our Hall bars with even lower diffusion coefficients $\left(71\right.$ and $46 \mathrm{~cm}^{2} / \mathrm{s}$ ) no saturation is observed. On the contrary, for similar values of diffusion coefficient in metals, low-temperature saturation of $\tau_{\phi}$ is frequently observed [3, 12]: this strongly suggests that the low temperature saturation of $\tau_{\phi}$ is not intrinsic and hence inconsistent with the GZ theory.

In conclusion, we have measured the disorder dependence of the phase coherence time in mesoscopic wires made from a two dimensional electron gas by varying the electronic diffusion coefficient over 2 orders of magnitude using an original ion implantation technique. We show that in the diffusive regime, the phase coherence time follows a power law as a function of the diffusion coefficient, $D^{\alpha}$, with $\alpha$ close to $1 / 3$, consistent with the standard model of decoherence proposed in Ref. [1]. When increasing the diffusion coefficient, the parameter $k_{F} l_{e}$ increases and samples become semi-ballistic; we then observe a new regime in which $\tau_{\phi}$ is independent of the diffusion coefficient.

We acknowledge helpful discussions with G. Montambaux, S. Kettemann, A. D. Zaikin, S. Florens, C. Strunk, and R. Whitney. Y. N. acknowledges financial support from the "JSPS Research program for Young Scientists". This work has been supported by the European Commission FP6 NMP-3 project 505457-1 "Ultra 1D" and the Agence Nationale de la Recherche under the grant ANR PNano "QuSpin".

[†] On leave from Tohoku University, Sendai, Japan.

[†] Present address: National Dong Hwa University, Taiwan.

[*] Mail to: bsm@listes.grenoble.cnrs.fr

[1] B. L. Altshuler et al., J. Phys. C 15, 7367 (1982).

[2] D. S. Golubev and A. D. Zaikin, Phys. Rev. Lett. 81, 1074 (1998); D. S. Golubev and A. D. Zaikin, Phys. Rev. B 74, 245329 (2006).

[3] P. Mohanty et al., Phys. Rev. Lett. 78, 3366 (1997).

[4] Y. Imry et al., Europhys. Lett. 47, 608 (1999).

[5] A. Zawadowski et al., Phys. Rev. Lett. 83, 2632 (1999).

[6] D. Natelson et al., Phys. Rev. Lett. 86, 1821 (2001).

[7] F. Pierre and N. O. Birge, Phys. Rev. Lett. 89, 206804 (2002).

[8] F. Schopfer et al., Phys. Rev. Lett. 90, 056801 (2003).

[9] F. Pierre et al., Phys. Rev. B 68, 085413 (2003).

[10] J. Wei et al., Phys. Rev. Lett. 96, 086801 (2006).

[11] C. Bäuerle et al., Phys. Rev. Lett. 95, 266805 (2005).

[12] F. Mallet et al., Phys. Rev. Lett. 97, 226804 (2006); T. A. Costi et al., Phys. Rev. Lett. 102, 056802 (2009).

[13] L. Saminadayar et al., Physica E 40, 12 (2007).

[14] Z. Ovadyahu, J. Phys. C: Solid State Phys. 16, L845 (1983).

[15] J. J. Lin and L. Y. Kao, J. Phys.: Condens. Matter 13, L119 (2001).

[16] M. Noguchi et al., J. Appl. Phys. 80, 5138 (1996).

[17] A. D. Wieck and K. Ploog, Surf. Sci. 229, 252 (1990)

[18] D. Diaconescu et al., Phys. Stat. Sol. (b) 245, 276 (2008).

[19] E. Akkermans and G. Montambaux, Mesoscopic physics of electrons and photons (Cambridge University Press, Cambridge, 2007).

[20] C. W. J. Beenakker and H. van Houten, Phys. Rev. B 38, 3232 (1988).

[21] B. Reulet et al., Europhys. Lett. 31, 305 (1995).

[22] Y. Lee et al., Phys. Rev. B 56, 9805 (1997) and references therein.

[23] B. N. Narozhny et al., Phys. Rev. B 65, 180202 (2002).

[24] H.-P. Wittmann and A. Schmid, J. Low Temp. Phys. 69, 131 (1987). 